\title{
Einblick ins Forschungslabor des Winterthurer Instituts für Gesundheitsökonomie - Teil II
}

\section{Sascha Hess, Klaus Eichler, Sylvia De Boni, \\ Marina Stähli, \\ Holger Auerbach}

Teil I des Beitrags ist erschienen in der SÄZ-Ausgabe 41/2011, S. $1567 f$.
Korrespondenz:

WIG Winterthurer Institut für Gesundheitsökonomie Prof. Dr. Klaus Eichler St. Georgenstrasse 70 CH-8401 Winterthur

\section{klaus.eichler(at)zhaw.ch} www.wig.zhaw.ch/

\begin{abstract}
Ausgangssituation
Seit dem 1. Juli 2009 gilt die revidierte eidgenössische Analysenliste (EAL) des Bundesamts für Gesundheit (BAG). Mit dieser EAL wurde die Höhe der Abgeltungen für ambulante Laborleistungen in der Schweiz nach KVG als behördlich erlassener Tarif neu festgelegt. Die EAL gilt für alle Erbringer von Laborleistungen, vom kleinen Labor einer Hausarztpraxis über die Labors von Spezialisten bis zu grossen Auftragslabors. Sie umfasst insgesamt über 1600 Positionen, wovon rund 170 in der Präsenzdiagnostik von Arztpraxislabors durchgeführt und abgerechnet werden können. Ziel der Revision der EAL durch das BAG war es, die Tarife der zunehmenden Automatisierung im Labor und dem technischen Fortschritt in der Analysentechnik anzupassen und betriebswirtschaftlich $\mathrm{zu}$ bemessen sowie sachgerecht auszugestalten.
\end{abstract}

\section{Zielsetzung unserer Untersuchung}

Die FMH hat zusammen mit der Ärztekasse Anfang 2011 das Winterthurer Institut für Gesundheitsökonomie (WIG) als unabhängige Institution beauftragt, eine Studie durchzuführen. In einem ersten Teil der Studie (SÄZ 41/2011) wurde geprüft, ob die Ziel-

\section{Abbildung 1}

Gegenüberstellung von Kosten und Vergütung. Dargestellt sind die jeweiligen empirisch erhobenen internen Kosten und die Vergütungen nach EAL bei den sechs untersuchten Analysen. Alle Angaben in CHF als Median.

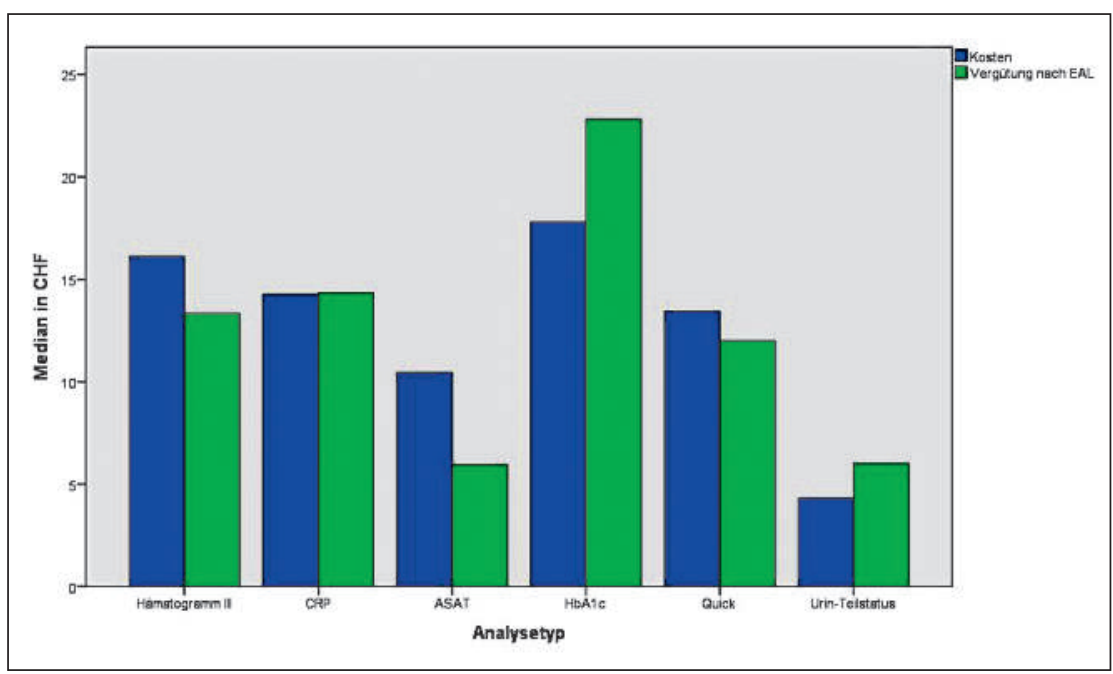

setzungen der Revision der EAL in Bezug auf die betriebswirtschaftliche Bemessung und die sachgerechte Struktur erreicht wurden. Im zweiten Teil der Studie wurde folgende Fragestellung wissenschaftlich untersucht: «Sind die Tarife der revidierten eidgenössischen Analysenliste kostendeckend für das ärztliche Praxislaboratorium?»

\section{Methodik}

Es wurde eine Prozesskostenanalyse unter Verwendung empirischer, prospektiv und retrospektiv erhobener Daten aus einer gewichteten Stichprobe von Praxislabors aus der Deutschschweiz durchgeführt. Die Praxislabors kamen aus den Fachbereichen Grundversorgung (Grundversorger Allgemeinärztinnen/-ärzte, Grundversorger/innen Allgemeine Innere Medizin), Gynäkologie, Pädiatrie, Kardiologie, Pneumologie und Gastroenterologie. Eingeschlossen waren alle Patienten, bei denen im Praxislabor eine Probenentnahme stattfand und eine oder mehrere der ausgewählten Laboranalysen im Praxislabor durchgeführt wurden. Unter Einbezug verschiedener Kriterien (z. B. Umsatzstärke, Häufigkeit der Durchführung) wurden folgende sechs Analysen für diese Studie ausgewählt: Hämatogramm III (H III; mittels automatisierter Methode), CRP (qn), ASAT, HbA1c, Quick/INR und UrinTeilstatus (5-10 Parameter).

Die Einheit der Auswertung war die einzelne Laboranalyse. Die Datenerhebung diente der Berechnung der praxisinternen Vollkosten pro Laboranalyse (Material-, Personal- und Fixkosten). Die Vollkosten wurden anschliessend dem vergüteten Tarif (inkl. Übergangszuschlag sowie Präsenztaxe und Suffixzuschlag bis zur tariflich festgelegten Obergrenze pro Konsultation) gegenübergestellt, um den Kostendeckungsgrad pro Analyse zu ermitteln.

Die Datenerhebung im Mai 2011 erfolgte mittels eines schriftlichen Erhebungsbogens durch die Medizinischen Praxisassistentinnen (MPAs) und Praxislaborbesitzer in den Praxislabors selbst.

\section{Ergebnisse}

Bei den 25 an der Studie teilnehmenden Praxislabors (Teilnehmerrate 7,5\%) aus der Deutschschweiz wurden bei den sechs ausgewählten Laboranalysen insgesamt 559 Messungen ausgewertet. Die Fachbereiche waren wie folgt vertreten: $60 \%$ Grundversorgung, 
Tabelle 1

Gegenüberstellung von Kosten und Vergütung (IQR= Interquartile range; $25 \%$ und $75 \%$ Perzentile).

\begin{tabular}{llll} 
Analyse & Kosten total & Vergütung total & $\begin{array}{l}\text { Differenz } \\
\text { Vergütung minus Kosten* }\end{array}$ \\
& CHF als Median (IQR) & CHF als Median (IQR) & $\begin{array}{l}\text { CHF als Median (IQR) } \\
\text { Hämatogramm III }\end{array}$ \\
& 16,1 & 13,3 & $-2,64$ \\
\hline CRP, qn & $(9,90 ; 20,4)$ & $(12,4 ; 14,0)$ & $(-7,03 ; 2,90)$ \\
\hline ASAT & 14,3 & 14,3 & 0,20 \\
& $(10,1 ; 18,1)$ & $(13,5 ; 15,0)$ & $(-4,28 ; 3,73)$ \\
\hline HbA1c & 10,4 & 5,94 & $-4,41$ \\
& $(7,26 ; 14,0)$ & $(5,38 ; 6,16)$ & $(-9,37 ;-1,58)$ \\
\hline Quick/INR & 17,8 & 22,8 & 3,53 \\
& $(7,05 ; 27,1)$ & $(21,4 ; 22,8)$ & $(-5,79 ; 15,8)$ \\
\hline Urin-Teilstatus & 13,4 & 12,0 & $-3,27$ \\
& $(12,5 ; 16,0)$ & $(10,0 ; 12,0)$ & $(-4,86 ;-1,30)$ \\
& 4,31 & 6,00 & 1,18 \\
\end{tabular}

*Vergütung minus Kosten: wegen der nicht normal verteilten Daten addieren sich die Mediane von «Kosten total» und «Vergütung minus Kosten» nicht exakt auf die Mediane von «Vergütungen total».

$24 \%$ Pädiatrie, $8 \%$ Gynäkologie, 4\% Kardiologie und $4 \%$ Pneumologie.

Die errechneten internen Kosten für die Analysenerstellung wurden den errechneten Vergütungen nach EAL im Praxislabor gegenübergestellt (Tabelle und Abbildung). Die Differenz aus Vergütung minus Kosten stellt einen positiven oder negativen Deckungsbeitrag dar, welcher in die betriebswirtschaftliche Rechnung des Praxisbetriebs einfliesst.

Dabei zeigt sich, dass auf Basis der uns vorliegenden Daten bei drei Analysen die Tarife der EAL zu keiner kostendeckenden Vergütung führen, und es resultiert somit ein negativer Deckungsbeitrag (Deckungsbeitrag pro Analysenerstellung: H III: -2,64 CHF; Quick/INR: -3,27 CHF; ASAT: -4,41 CHF).

Bei zwei Analysen sind die Tarife der EAL kostendeckend, und es resultiert ein positiver Deckungsbeitrag (Deckungsbeitrag pro Analysenerstellung: UrinTeilstatus: +1,18 CHF; HbA1c: +3,53 CHF).

Bei einer Analyse sind die Tarife der EAL kostendeckend und entsprechen ziemlich genau den empirisch ermittelten Erstellungskosten im Praxislabor (Deckungsbeitrag pro Analysenerstellung: CRP: $+0,20 \mathrm{CHF})$.

Den überwiegenden Kostenanteil bei den meisten Analysen stellen die Materialkosten dar, mit besonderer Ausprägung beim HbA1c. Bei dieser Analyse ist der Testträger relativ teuer. Beim Hämatogramm III fallen dagegen die Gerätekosten und die Personalkosten deutlicher ins Gewicht als bei den anderen Analysen. Die Ergebnisse variieren bei den durchgeführten Sensitivitätsanalysen, welche verschiedene Annahmen auf der Kosten- und auf der Ertragsseite überprüften, nur geringfügig.

\section{Diskussion}

Die Studie hat aktuelle Daten aus der Versorgungslandschaft der Praxislabors erhoben und somit einen Beitrag zu mehr Transparenz geleistet. Die erhobenen Praxislaborkosten stellen einen konservativen Schätzwert dar, da extrem nach oben abweichende Kostendaten durch die Verwendung des Medians bei der Auswertung weitgehend ausgeklammert wurden. Als methodische Limitation ist vor allem die niedrige Teilnahmerate zu nennen.

Auf Basis der vorliegenden Daten zeigt sich, dass drei von sechs untersuchten Laboranalysen im Praxislabor nicht kostendeckend mit der revidierten EAL vergütet werden. Dies kann zu unterschiedlichen Anreizsituationen bei der Erstellung von Laboranalysen führen.

Welche definitiven Auswirkungen dies für die Zukunft des Praxislabors, die Situation von Auftragslabors sowie für die Qualität der Patientenversorgung hat, wird aktuell diskutiert. 